Journal of Applied Pharmaceutical Science Vol. 5 (06), pp. 133-137, June, 2015

Available online at http://www.japsonline.com

DOI: $10.7324 /$ JAPS.2015.50622

ISSN 2231-3354 (cc) BY-NC-SA

\title{
Drug-like properties of potential anti-cancer compounds from Cameroonian flora: A virtual studies
}

\author{
Ibezim Akachukwu $^{1 *}$, Onyia Kelechi ${ }^{2}$,Ntie-Kang Fidele ${ }^{3}$, Nwodo Ngozi Justina ${ }^{1}$ \\ ${ }^{1}$ Department of Pharmaceutical and Medicinal Chemistry, University of Nigeria, Nsukka 410001, Nigeria. \\ ${ }^{2}$ Department of Pure and Industrial Chemistry, University of Nigeria, Nsukka 410001, Nigeria. \\ ${ }^{3}$ Chemical and Bioactivity Information Centre, Department of Chemistry, University of Buea, Buea, Cameroon.
}

\section{ARTICLE INFO \\ Article history: \\ Received on: 11/01/2015 \\ Revised on: 28/01/2015 \\ Accepted on: 19/04/2015 \\ Available online: $27 / 06 / 2015$}

\section{Key words:}

Cancer, Natural products,

Drug-likeness, Physico-

chemical properties.

\begin{abstract}
Assessment of the 'drug-like' using a set of calculated molecular descriptors, of a collection of 195compounds from plants in Cameroon exhibiting in vitro or/and in vivo activities against various cancer cell lines was made. Lipinski's 'Rule of Five' (Ro5) was used to evaluate drug-likeness/oral availability of the compounds by making use of popular parameters like Molecular Weight (MW), predicted lipophilicity (log $P$ ), number of Hydrogen Bond Donors/Acceptors (HBD and HBA) and Number of Rotatable Bonds (NRB). The drug-like properties of the studied compounds were compared to that of the Dictionary of Natural Products (DNP). Our results revealed that $79.50 \%, 43.60 \%$ and $12.30 \%$ of our dataset fall within the recommended range for 'drug-like', 'lead-like' and 'fragment-like' respectively with significant enhancement of some oral availability parameters over DNP and this paints the significant of our dataset in search for lead-like anti-cancer molecules with desirable 'drug-like' properties.
\end{abstract}

\section{INTRODUCTION}

Cancer is a term used to refer to all malignant tumors. A neoplasm or tumor is an abnormal mass of tissue, the growth of which exceeds and is uncoordinated with that of the normal tissue and continues in the same manner after cessation of the stimuli which have initiated it (Ashutosh, 2007). Cancer is the second most common disease-related cause of death of human population (Gibbs, 2000). It is known to be responsible for about $\sim 7.6$ million deaths $(\sim 13 \%$ of all deaths $)$ worldwide according to the World Health Organization (WHO, 2013). In spite of the enormous efforts and progress in the field of cancer research, the WHO estimates that the threatening of cancer disease will worsen (GLOBOCAN, 2008). Plant materials (and their derived photochemical) have been extensively used as chemotherapy to treat various forms of cancer and several reviews on medical plants used in the treatment of cancer and tumor have been published(Koehn et al., 2005; Hartwell, 1970; Graham et al., 2010; Rahman et al., 2013; Cragg and Newman, 2003). A report

* Corresponding Author

Ibezim Akachukwu, Department of Pharmaceutical and Medicinal

Chemistry, University of Nigeria, Nsukka. 410001 Nigeria.

Mobile: +2348038032 676, E-mail: saintakaben@yahoo.com by 1990 has it that about $80 \%$ of drugs were either Natural Products (NPs) or analogues inspired by them. According to a recent review on new chemical entities by Newman and Cragg, $\sim 49 \%$ of anticancer drugs were either natural products or natural product-related synthetic compounds or their mimetics (Newman and Cragg, 2010). These data are coherent with a previous report by the same authors, which showed $47 \%$ of a total of 155 anticancer drugs approved up to 2006 were either natural products or directly derived from NPs lead compounds by semi-synthesis (Newman and Cragg, 2007). Cameroonian medicinal plants are endowed with NPs with intriguing chemical structures and promising biological activities (Pieme et al., 2012; Ndonsta et al., 2011). Many drugs with very interesting properties often fail to enter the market after much labor and investment, as a result of poor performance in physico-chemical assessment assays (Darvas et al., 2002), it has become customary to assess the 'drug-likeness' and physic-chemical properties of potential drug candidates early enough to cut down cost (DiMasi et al., 2003). This is often carried out by the use of experimental 'wet' screens or by computer-based (in silico) predictions using calculated molecular descriptors. The latter is much faster and less costly, making use of experimental data, which had accumulated by the close of the 1990s (Hodgson, 2001). 
In silico methodologies make use of quantitative structure-activity relationships (QSARs) or knowledge-based approaches, using computer software (Button et al., 2003). In the current study, an attempt has been made to evaluate how 'drug-like' naturally occurring anticancer compounds are, by analyzing computed molecular descriptors for 195 anticancer compounds from Cameroonian medicinal plants and comparing them with that of DNP. This work is a follow up of our continuous development of NP libraries and in silico analysis of the 'drug-likeness' and physic-chemical profile, with the view of drug discovery (NtieKang et al., 2013a; 2013b; 2013c; 2013d; 2013e; 2014).

\section{MATERIALS AND METHODS}

\section{Data Sources}

The chemical structures of pure compounds as well as their biological activities were retrieved from literature sources comprising mainly published articles from across the major journals of natural product chemistry. The exact chemical structures were confirmed from Dictionary of Natural Products.

\section{Generation of 3D Models, Optimization and Calculation of Molecular Descriptors}

Based on the known chemical structures of the NPs, all 3D molecular structures were generated using the graphical user interface (GUI) of the MOE software (Chemical Computing Group Inc., 2010)running on a Linux workstation with a $3.5 \mathrm{GHz}$ Intel Core2 Duo processor.

The 3D structures were generated using the builder module of MOE and energy minimization was subsequently carried out using theMMFF94 force field (Halgren, 1996) until a gradient of $0.01 \mathrm{kcal} / \mathrm{mol}$ was reached. The $3 \mathrm{D}$ structures of the compounds were then saved as in pdb format included into a MOE database (.mdb) file which is suitable for use in several virtual screening workflow protocols. The Molar Weights (MW), Number Rotatable Bonds (NRB), lipophilicity ( $\log P$ ), Hydrogen Bond Acceptor (HBA), Hydrogen Bond Donor (HBD), and Lipinski violations were calculated using the molecular descriptor calculator included in the QuSAR module of the MOE package.

\section{RESULTS AND DISCUSSION}

The mean values for the relevant descriptors used to evaluate the 'drug-likeness' have been shown in table 1 for total compound library, as well as the standard 'drug-likeness', leadlike' and fragment-like' libraries. The concepts 'drug-likeness', lead-likeness' and 'fragment-likeness' have been respectively defined following the criteria for $\mathrm{MW}, \log P, \mathrm{HBA}$, HBD and NRB: $(\mathrm{MW}<500, \log \mathrm{P}<5, \quad \mathrm{HBD} \leq 5, \quad \mathrm{HBA} \leq 10)$ (Lipinski et al., 1997); (150 $\leq \mathrm{MW} \geq 350, \log \mathrm{P} \leq 4, \mathrm{HBD} \leq 3, \mathrm{HBA} \leq 6)$ (Teague et al., 1999; Oprea, 2002; Schneider, 2002) and $(\mathrm{MW} \leq 250,-2 \leq \log P \leq 3, \mathrm{HBD}<3, \mathrm{HBA}<6$ and $\mathrm{NRB}<3$ ) (Verdonk et al., 2003). This respectively gave 99, 66, and 23 compounds in the drug-like, lead-like and fragment-like subsets.
Table 1: Summary of mean physic-chemical properties distributions of the library in comparison with the various subsets.

\begin{tabular}{ccccc}
\hline & Total & Drug-like & Lead-like & Fragment-like \\
\hline Lib size & 195 & 155 & 85 & 24 \\
MW & 4.7 .37 & 339.83 & 279.20 & 203.27 \\
Lon P & 3.96 & 2.68 & 2.29 & 1.90 \\
HBA & 5.96 & 4.99 & 4.43 & 3.88 \\
HBD & 2.35 & 1.89 & 1.59 & 1.19 \\
NRB & 5.31 & 2.32 & 1.96 & 1.13 \\
\hline
\end{tabular}

\section{Lipinski's test for oral availability}

Lipinski's 'rule of five' Ro5 has often been used as an assessment criterion for oral availability, although this rule sometimes does not apply to NPs (Lipinski, 2000; Quinn, 2008). It was observed that $78.97 \%$ of the studied compounds complied with the MW criterion (MW<500Da), while respectively $70.77 \%$, $91.28 \%$ and $93.33 \%$ respected the $\log P<5, \mathrm{HBA} \leq 10$ and $\mathrm{HBD} \leq 5$ parameters (Fig. 1). In addition, $72.82 \%$ of the compounds respected the criterion of the NRB, a rule often added to Lipinski's Ro5 to take drug metabolism and pharmacokinetics (DMPK) profiles into consideration. This globally showed that 155 of the compounds respected the Ro5, hence constituting the 'drug-like' subset. Meanwhile $72.82 \%$ of the compounds in the total library showed $\leq 2$ violation of the Ro5 (Fig. 1F). The histogram of the variation of the MW parameter showed a peak between 300 and $400 \mathrm{Da}$ (fig. 1A). The computed $\log P$ values were within reasonable limits, with only values for five compounds exceeding 10 units (fig. 1B). The $\log P$ distribution showed a rough Gaussian curve, with a peak centered at $\log P=2$ unit (fig. 1B), while the HBA and HBD parameters both showed a rapid increase to peak values of 46 and 56 at four acceptors and three donor respectively (fig. 1C and 1D). Both curves fell rapidly to 13 acceptors and 6 donors respectively for the bulkiest compounds. The curve of NRB (fig. 1E) showed a peak values at zero rotatable bonds (RBs) with the bulkiest NPs having as many as 30 RBs.

The scattered plots (fig. 2) revealed that the regions of highest population densities fall within the 'Lipinski region of interest', i.e., $\mathrm{MW}<500,-2<\log \mathrm{P}<5, \mathrm{HBA} \leq 10, \mathrm{HBD} \leq 5$ and for which $\mathrm{NRB}<5$.

\section{Comparison with the Dictionary of Natural Products (DNP)}

A comparison of the anticancer NP library has been carried out with the much larger Dictionary of Natural Products (DNP), containing 126,140 compounds (Dictionary of natural products, 2005). In our discussion, emphasis has been laid on the Lipinski compliance zones $(\mathrm{MW}<500,-2 \leq \log \mathrm{P}<5, \mathrm{HBA}<10$, and $\mathrm{HBD}<5)$, the values being expressed as percentage counts of the respective data sets. The distributions of the individual parameters for the two datasets have been shown in Fig. 3. These histograms generally show an enhancement of the distributions of the anticancer dataset over the DNP for Lipinski properties. The MW distribution histograms (Fig. 3A) showed that both curves peak at 301-400 Da. However, the anti-cancer dataset has higher percentage abundance for the regions301-400 and 401-500 Da within the Lipinski compliance zone with an enhancement of $9.20 \%$ and $2.33 \%$ respectively in MW. Below this range, the 
percentages were reduced for our dataset, when compared to the DNP. The proportions of the two databases that satisfy Lipinski's MW property $(<500 \mathrm{Da})$ were about $78.98 \%$ for our dataset and lower $(74.00 \%)$ for the DNP dataset. The maximum values for the $\log P$ distributions were both at $2.5 \log P$ units (Fig. 3B). The anticancer dataset is enhanced over the DNP for $1.0<\log \mathrm{P}<5$ by $13.46 \%$ and a $25.70 \%$ enhancement of the DNP over the anticancer dataset for $-2<\log \mathrm{P}<1.0$. The HBA and HBD distributions (Fig. 3C-D) of the anti-cancer and DNP datasets respectively showed an interesting result. The peak of distributions


Violaton of Lipinski's Ro5 for the HBD for the two datasets is situated at zero and two donors for DNP and anti-cancer datasets (22.50\% and 28.72\%) respectively. Similarly, the peak of distributions for HBA for the two datasets is both situated at four at varying percentages of $14.15 \%$ and $23.59 \%$ for DNP and anti-cancer datasets respectively. The overall summary of the four Lipinski parameters for the two datasets thus reveals that the anti-cancer dataset library is more 'drug-like' than the DNP, indicating that the chances of finding 'lead-like' molecules with improved DMPK properties within our library dataset are quite significant.
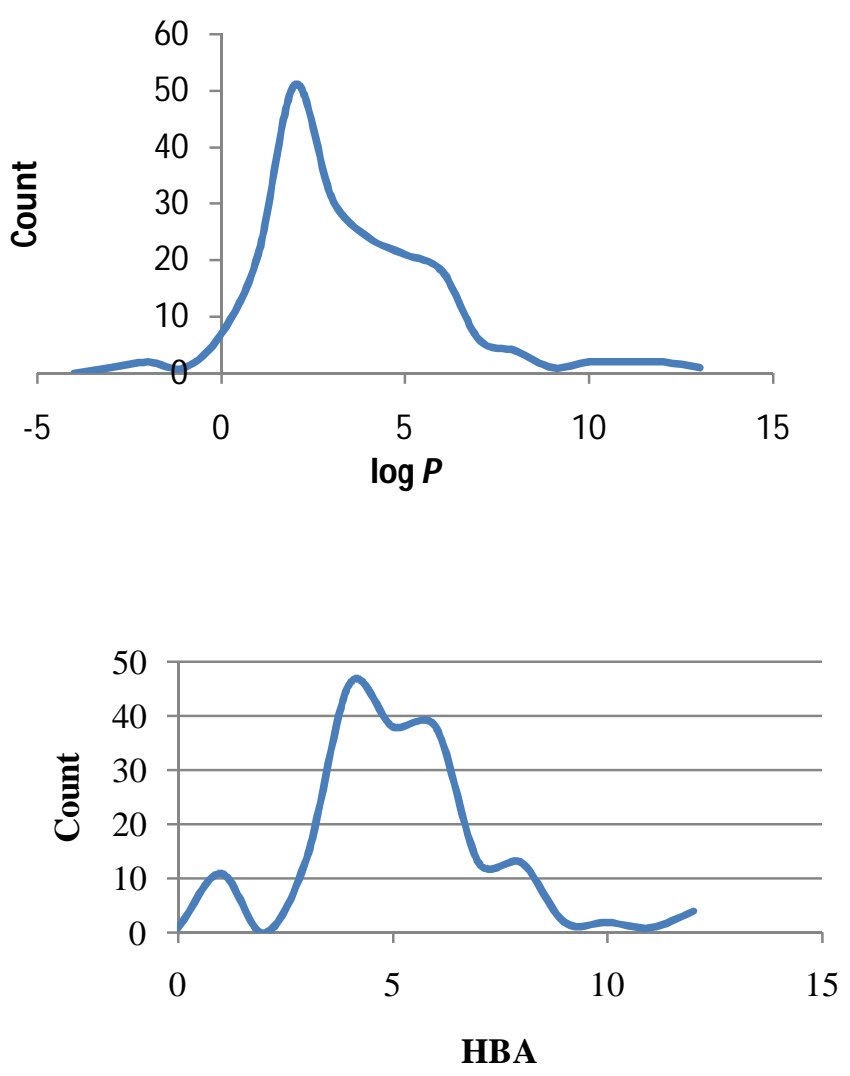

Fig. 1: Distribution of physico-chemical properties used to assess Lipinski's Ro5: A MW, Blog $P, \mathbf{C}$ HBD, DHBA, ENRB, and FLipinski's violation. 



Fig. 2: 2D scattered plot showing pair wise distribution of 'drug-likeness' descriptors; A MW against $\log P$, BMW against HBD, CMW against HBA and DMW against NRB.
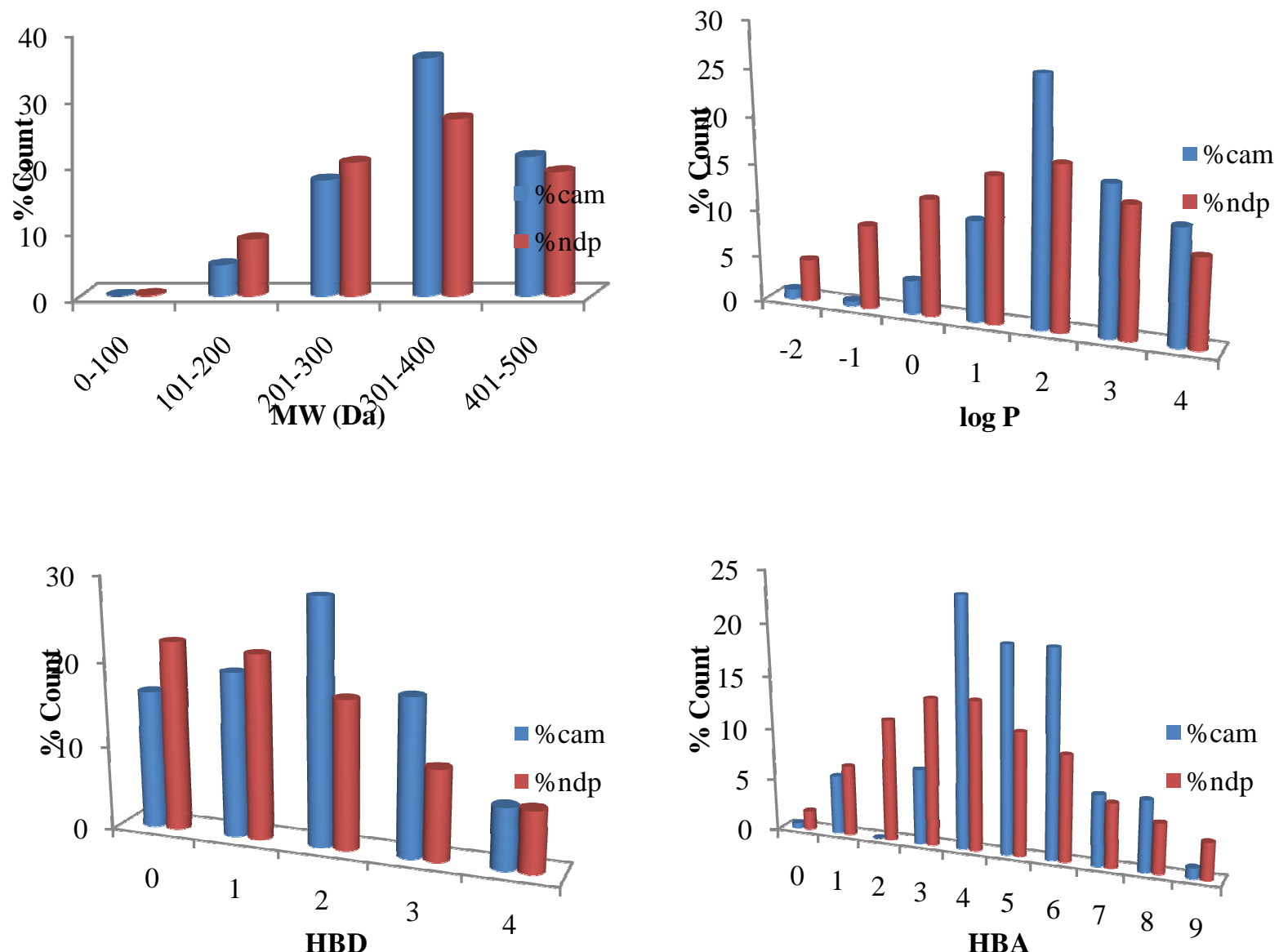

Fig. 3: Comparison of property distribution for the two datasets by percentage distributions; A MW, B $\log P, \mathbf{C}$ HBD and $\mathbf{D}$ HBA. For substructure B, the $x$-axis label is the lower limit of binned data, e.g. 0 equivalents to 0 to 1. 


\section{CONCLUSION}

The chances of identifying suitable compounds could be further enhanced if the search library presents reasonable level of 'drug-likeness' and 'lead-likeness'. In this study, it could be inferred, from the ranges of computed molecular descriptors, that our library contains naturally occurring anti-cancer compounds with interesting 'drug-like' and physico-chemical properties rendering it a good starting point for NPs based drug discovery projects. Hence, we hope to go further to evaluate the potentials of the compounds as inhibitors of various anti-cancer drug-targets.

Acknowledgments: Technical support is acknowledged from Chemical and Bioactive Information Centre, University of Buea, for using the MOE software.

\section{REFERENCE}

Ashidi JS, Houghton PJ, Hylands PJ, Efferth T. Ethnobotanical survey and cytotoxicity testing of plants South-western Nigeria used to treat cancer, with isolation cytotoxic constituents from Cajanus cajan Millsp. Leaves. J Ethnopharmacol. 2010; 128:501-512.

Ashutosh K. 2007. Medicinal Chemistry. Delhi, India: New Age International Ltd.

Button WG, Judson PN, Long A, Vessey JD. Using absolute and relative reasoning in the prediction of the potential metabolism of xenobiotics.J Chem Inf Comput Sci. 2003; 43:1371-1377.

Chemical Computing Group Inc. Molecular Operating Environment Software. Montreal; 2010.

Cragg GM, Newman DJ. Plants as a source of anti-cancer and anti-HIV agents. Ann Appl Biol. 2003; 143:127-133.

Darvas F, Keseru G, Papp A, Dormán G, Urge L, Krajcsi P. In silico and ex silico ADME approaches for drug discovery.Curr Top Med Chem. 2002; 2:1287-1304.

Dictionary of natural products on CD-rom. Chapman and Hall/CRC Press, London 2005.

DiMasi JA, Hansen RW, Grabowsk HG. The price of innovation: new estimates of drug development costs. J Health Econ. $2003 ; 22: 151-185$.

Gibbs JB. Mechanism-based target identification and drug discovery in cancer research. Science, 2000; 287:1969-1973.

GLOBOCAN 2008. Available at:
http://globocan.iarc.fr/factsheets/populations/asp?uno=900[Accessed 04 August 2014].

GrahamJG, Quinn ML, Fabricant DS, Farnsworth NR. Plants used against cancer- an extension of the work of Jonathan Hartwell.J Ethnophamacol. 2010; 73:347-377.

Halgren TA. Merck molecular force field. J Comput Chem. 1996; 17:490-641. $33: 97-425$

Hartwell J. Plant used against cancer. A survey.Lloydia.1970;

Hodgson J. ADMET-turning chemicals into drugs. Nat Biotechnol. 2001; 19:722-726.

KoehnFE, Carter GT. The evolving role of natural products in drug discovery. NatRevDiscov. 2005; 4:206-220.

Lipinski CA. Drug-like properties and the causes of poor solubility and poor permeability. J Pharmacol Toxicol Methods, 2000; 44:235-249.

Lipinski CA, Lombardo F, Dominy BW, Feeney PJ. Experimental and computational approaches to estimate solubility and permeability in drug discovery and development settings. Adv Drug Deliv Rev. 1997; 23:3-25.
Ndonsta BL, Tatsimo JSN, Csupor D, Forgo P, Berkecz R, Bere'nyi A, Tene M, Molna'r J, Zupko' I, Hohmann J, Tane P. Alkylbenzoquinones with antiproliferative effect against human cancer cell lines from stem of Ardisia kivuensis. Phytochem Lett. 2011; 4:227230 .

Newman DJ, Cragg GM. Natural products as source of new drugs over the last 25 years. J Nat Prod. 2007; 70:461-477.

Newman DJ, Cragg GM. Natural Products As Sources of New Drugs over the 30 Years from 1981 to 2010.J Nat Prod. 2010; 75:311-335.

Ntie-Kang F. An in silico evaluation of the ADMET profile of the streptome DB database. Springer Plus, 2013a; 2:353.

Ntie-Kang F, Lifongo LL, Mbah JA, Owono Owono LC, Megnassan E, Mbaze LM, Judson PN, Sippl W, Efange SMN. In silico drug metabolism and pharmacokinetic profiles of natural products from medicinal plants in the Congo basin. In silico Pharmacol. 2013b; 1:12.

Ntie-Kang F, Mbah JA, Lifongo LL, Owono Owono LC, Megnassan E, Mbaze LM, Judson PN, Sippl W, Efange SMN. Assessing the pharmacokinetic profile of the CamMedNP natural products database: an in silico approach. Org Med Chem Lett. 2013c; 3:10.

Ntie-Kang F, Mbah JA, Mbaze LM, Lifongo LL, Scharfe M, Ngo Hanna J, Cho-Ngwa F, Amoa Onguéné P, Owono Owono LC, Megnassan E, Sippl W, Efange SMN. CamMedNP: building the Cameroonian 3D structural natural products database for virtual screening. BMC Complement Altern Med. 2013d; 13:88.

Ntie-Kang F, Zofou D, Babiaka SB, Meudom R, Scharfe M, Lifongo LL, Mbah JA, Mbaze LM, Sippl W, Efange SMN. AfroDb: a select highly potent and diverse natural product library from African medicinal plants. PLoS ONE 2013e; 8, e78085. doi:10.1371/journal.pone.0078085.

Ntie-Kang F, Nwodo NJ,Ibezim A, Simoben CV, Karaman B,Ngwa FV, Sippl W, Adikwu MU, Mbaze ML. Molecular Modeling of Potential Anticancer Agents from AfricanMedicinal Plants. J Chem Inf Model. 2014; 54:2433-2450.

Oprea TI. Current trends in lead discovery: are we looking for the appropriate properties? J Comput Aided Mol Des. 2002; 16:325-334.

Pieme AC, Ngogang J, Costache M. In vitroantiproliferative and anti-oxidant activities of methanol extracts of Urena lobata and Viscum album against breast cancer cell lines. Toxicol and Environ Chem. 2012;94:987-999.

Quinn RJ, Carroll AR, Pham MB, Baron P, Palframan ME, Suraweera L, Pierens GK, Muresan S. Developing a drug-like natural product library. J Nat Prod. 2008; 71:464-468.

Rahman MM, Khan MA. Anti-cancer potential of South Asian plants. Nat Prod Bioprospect. 2013; 3:74-88.

Schneider G. Trends in virtual computational library design. Curr Med Chem. 2002; 9:2095-2102.

Teague SJ, Davis AM, Leeson PD, Opea TI. The design of leadlike combinatorial libraries. Angew Chem Int Ed. 1999; 38:3743-3748.

Verdonk ML, Cole JC, Hartshorn ML, Murray CW, Taylor RD. Improved protein-ligand docking using GOLD. Proteins, 2003; 52:609_ 623.

WHO media centre 2013.Available at: http://www.who.int/mediacentre/factsheets/fs297/en/index.html[Accessed 04 Febuary 2014]

How to cite this article:

Ibezim, Akachukwu, Onyia, Kelechi, Ntie-Kang, Fidele,Nwodo, Ngozi Justina. Drug-like properties of potential anti-cancer compounds from Cameroonian flora: A virtual studies. J App Pharm Sci, 2015; 5 (06): 133-137. 